D]

'School of Public Health, al-Farabi Kazakh National University, Almaty, Kazakhstan.

2Dept of Paediatrics, "Sapienza" University of Rome, Rome, Italy.

${ }^{3}$ Dept of Respiratory Medicine, University Hospital, Besançon, France.

${ }^{4}$ Dept of Physiology, University Hospital, Besançon, France.

Cite as: Vinnikov D, Nenna $R$, Soumagne T. Will an electronic nose help at high altitude? Breathe 2018; 14: 322-324.

\title{
Will an electronic nose help at high altitude?
}

\section{Landmark papers in respiratory medicine}

Travel and stay at high altitude for recreational purposes or work is challenging, because the demand for acute acclimatisation to hypobaric hypoxia triggers distinct respiratory, cardiovascular, neurohumoral and psychological shift. The respiratory response, such as increase in respiratory rate, hypoxic vasoconstriction and other mechanisms, may be most pronounced. Acute mountain sickness (AMS) is likely to develop in unacclimatised subjects who rapidly ascend to high altitude, with clearly growing prevalence with increasing altitude [1]. Because AMS is diagnosed when other potential reasons are ruled out, the diagnosis is to a great extent subjective, because the leading symptom of this condition is headache. This article aims to provide more information on AMS, a prevalent condition at high altitude, as well as on emerging novel methods of diagnosis, and to discuss the feasibility of their use at high altitude.

AMS diagnosis in the field may be easier with conventional scales, such as the Lake Louise Score (LLS), when a given cut-off score makes the diagnosis very likely, calling for an intervention, which depends on the severity and maximum altitude. Whether one uses the LLS or the Environmental Symptoms Questionnaire (ESQ) III (which is slightly different), or even other scales, some disagreement and uncertainty over the diagnosis may still remain; therefore, the search for other reliable ways to diagnose AMS is ongoing [2]. In a recent study at Capanna Regina Margherita (Italy) at $4554 \mathrm{~m}$ above sea level, BeRENDSEN et al. [3] tested the hypothesis that analysing the traces of exhaled volatile organic compounds (VOCs) could help distinguish between $\mathrm{AMS}^{+}$and $\mathrm{AMS}^{-}$subjects. The rationale for such an approach is based on the assumption that oxidative stress is a part of AMS pathophysiology and that AMS is accompanied by a metabolic stress, which together result in a change in VOC exhaled concentrations. They used an electronic nose, which does not simply detect the concentrations of selected VOCs in the exhaled air, but also verifies the cumulative signature of the overall VOC concentration. This technology is novel, but its potential for use in high-altitude medicine to help diagnose AMS is promising.

With a given device, Aeonose (The eNose Company, Zutphen, the Netherlands), and a sample size of 101 climbers, Berendsen et al. [3] failed to distinguish between $\mathrm{AMS}^{+}$and $\mathrm{AMS}^{-}$subjects, since logistic regression showed no difference in the artificial neural network predicted values between the two groups. The authors listed five possible explanations for this outcome. One of these is an assumption that VOC, in fact, do not change in AMS, while another could be the absence of rigid AMS diagnosis criteria. Interestingly, the discrepancy between two conventional scales in this study was but verifies the cumulative signature, reflecting the overall VOC concentration. This technology is novel but promising. http://ow.ly/IPCt30mblBV 
quite high, as concordance between LLS $\geq 3$ and abbreviated ESQ (ESQc) $\geq 0.7$ was only 0.41 ( $95 \% \mathrm{Cl}$ 0.25-0.57), with some increase after the exclusion of a sleep item from LLS.

This study by BERENDSEN et al. [3] may have clear practical implications. First and foremost, it adds more emphasis to the need to plan and conduct studies to test further biochemical or other instrumental ways to diagnose AMS. Several instruments focusing on different clinical features have been proposed, but a single reliable gold standard for diagnosing AMS is still lacking. Instruments such as hypoxic cardiopulmonary exercise testing represent appealing tools and are based on compelling evidence for the diagnosis of subsequent AMS [4].

Secondly, this study reminds us that a coherent explanation of AMS physiopathology is still lacking. Indeed, AMS is thought to be multifactorial and not to rely on a single pathway. Responses of the body to hypoxia involve several hypoxiainducible factors, which have multiple functions throughout the physiological spectrum such as angiogenesis and nitric oxide metabolism [5]. The rationale of the study by BERENDSEN et al. [3] is the fact that oxidative stress in response to hypoxia may contribute to AMS. Indeed, altered redox homeostasis has been suggested in the physiopathology of AMS with subsequent disordered cerebral autoregulation [6]. Measuring VOCs as a surrogate of oxidative stress is therefore an interesting way to assess AMS, since the concentration of a volatile substance in exhaled breath is a reflection of its concentration in blood. However, as stated by the authors, this given device was probably not sensitive enough or the algorithm used in it was not good enough for AMS, as opposed to diabetes or other conditions, for which it had been previously tested. Effort should be made to improve this device, as a strong rationale supports it. Another possible explanation for the lack of distinction between $\mathrm{AMS}^{+}$and $\mathrm{AMS}^{-}$subjects in this study is that oxidative stress is not the sole cause of disordered cerebral autoregulation. Highaltitude illness, including AMS, is largely driven by genetic factors and inter-individual variation is marked among studies on AMS.

AMS is not life-threatening and can be treated at altitude; however, it may hamper habitual functioning and further ascent, and can even be incapacitating. Many efforts have been tailored to verify risk factors for this condition, and rate of ascent and altitude reached [1] are strongly associated with the risk, whereas studies of other

\section{Self-evaluation question}

The use of electronic nose technology in AMS is sensible because:

a) AMS is an acute condition at altitude and headache is a leading symptom

b) VOC measurement in the exhaled air is a surrogate of oxidative stress, and hypoxia may promote such stress

c) VOC measurement in the exhaled air helps differentiate between mild and severe AMS

d) Cigarette smoking is the leading risk factor for AMS

predictors, such as age [7] or smoking [8], have not yet yielded consistent findings.

Slow or graded ascent is the best way to prevent AMS, and those with chronic conditions should be thoroughly evaluated before starting. Current guidelines on AMS prevention and treatment presume graded ascent and the use of acetazolamide or dexamethasone for prevention [1]. Doctors are also advised to grade travellers as having low, moderate or high risk of AMS, where the major criteria for shifting to higher risk are rapid ascent or any prior history of AMS. In general, those classified as low-risk subjects should refrain from taking acetazolamide and ascent gradually. AMS treatment requires more vigorous intervention, including evacuation (descent to lower altitude), the use of oxygen, a portable hyperbaric chamber (Gamow chamber), acetazolamide or dexamethasone [1]. Although descent is the most effective way to ameliorate AMS symptoms, it is not always possible, especially in extreme altitude climbers; therefore, the decision should be made as to which medication should be used on top of supplemental oxygen.

Following this study by BERENDSEN et al. [3], we believe that the use of electronic technology is very promising in remote high-altitude settings, when adapted and further developed. Electronic technology, such as an electronic nose to "sniff" AMS, may help doctors differentiate between other causes of similar symptoms and target specific treatment to such patients, including oxygen, acetazolamide or even evacuation in cases of clinical deterioration. Despite distinct limitations [9, 10], the technology is likely to develop in the future. The use of such a device could have a significant impact on AMS detection, not only for leisure mountaineering but also in occupational settings.

\section{Conflict of interest}

None declared. 


\section{Suggested answer}

b.

\section{References}

1. Luks AM, Mclntosh SE, Grissom CK, et al. Wilderness Medical Society practice guidelines for the prevention and treatment of acute altitude illness: 2014 update. Wilderness Environ Med 2014; 25: Suppl. 4, S4-S14.

2. Meier D, Collet TH, Locatelli I, et al. Does this patient have acute mountain sickness? The rational clinical examination systematic review. JAMA 2017; 318: 1810-1819.

3. Berendsen RR, van Vessem ME, Bruins M, et al. Electronic nose technology fails to sniff out acute mountain sickness. High Alt Med Biol 2018; 19: 232-236.

4. Canouï-Poitrine F, Veerabudun K, Larmignat $\mathrm{P}$, et al. Risk prediction score for severe high altitude illness: a cohort study. PLoS One 2014; 9: e100642.

5. West JB. High-altitude medicine. Am J Respir Crit Care Med 2012; 186: 1229-1237.
6. Bailey DM, Evans KA, James PE, et al. Altered free radical metabolism in acute mountain sickness: implications for dynamic cerebral autoregulation and blood-brain barrier function. J Physiol 2009; 587: 73-85.

7. Wu Y, Zhang C, Chen Y, et al. Association between acute mountain sickness (AMS) and age: a meta-analysis. Mil Med Res 2018; 5: 14

8. Vinnikov D, Blanc PD, Steinmaus C. Is smoking a predictor for acute mountain sickness? Findings from a meta-analysis. Nicotine Tob Res 2016; 18: 1509-1516.

9. Boots AW, Bos LD, van der Schee MP, et al. Exhaled molecular fingerprinting in diagnosis and monitoring: validating volatile promises. Trends Mol Med 2015; 21: 633-644.

10. Horváth I, Barnes PJ, Loukides S, et al. A European Respiratory Society technical standard: exhaled biomarkers in lung disease. Eur RespirJ 2017; 49: 1600965. 